\title{
Non-sexual HPV transmission and role of vaccination for a better future (Review)
}

\author{
AIDA PETCA $^{1,2^{*}}$, ANDREEA BORISLAVSCHI $^{2^{*}}$, MONA ELENA ZVANCA $^{1,2^{*}}$, RAZVAN-COSMIN PETCA $^{1,3}$, \\ FLORICA SANDRU ${ }^{1,4}$ and MIHAI CRISTIAN DUMITRASCU ${ }^{1,5}$
}

\footnotetext{
${ }^{1}$ 'Carol Davila' University of Medicine and Pharmacy, 050474 Bucharest; ${ }^{2}$ Department of Obstetrics and Gynecology, Elias Emergency University Hospital, 011461 Bucharest; ${ }^{3}$ Department of Urology, 'Prof. Dr. Th. Burghele' Clinical Hospital, 050659 Bucharest; ${ }^{4}$ Department of Dermatology, Elias Emergency University Hospital, 011461 Bucharest;

${ }^{5}$ Department of Obstetrics and Gynecology, University Emergency Hospital, 050098 Bucharest, Romania
}

Received July 6, 2020; Accepted August 5, 2020

DOI: $10.3892 / \mathrm{etm} .2020 .9316$

\begin{abstract}
Human papilloma viruses (HPVs) belong to the Papillomaviridae family and are epitheliotropic infecting squamous epithelia (skin and mucosae). HPV is estimated to be the cause of $99 \%$ of cervical cancers (there is no evidence of significant genetic predisposition for cervical cancer), $90 \%$ of anal cancer, $65 \%$ vaginal cancers, $50 \%$ vulvar cancers, and 45-90\% oropharyngeal cancers. The route of HPV transmission is primarily through skin-to-skin or skin-to-mucosa contact. Sexual transmission is the most documented, but there have been studies suggesting non-sexual courses. The horizontal transfer of HPV includes fomites, fingers, and mouth, skin contact (other than sexual). Self-inoculation is described in studies as a potential HPV transmission route, as it was certified in female virgins, and in children with genital warts (low-risk HPV) without a personal history of sexual abuse. Vertical transmission from mother to child is another HPV transfer course. Several studies have emphasized the possibility of infection through the amniotic fluid, or the placenta, or via contact with maternal genital mucosa during natural birth. Waterborne transmission of HPV has never been demonstrated; however, HPV DNA has been detected in water environments. Routine hygiene measures are proven to be inefficient in preventing HPV transmission, as the studies which have evaluated samples of HPV on contaminated medical equipment (after standard disinfection) have found them to be still positive. Annual costs associated with the morbidity and mortality of HPV-related diseases are estimated at approximately $\$ 4$ billion. Once the HPV vaccine program in Australia was launched, many studies reported the
\end{abstract}

Correspondence to: Dr Razvan-Cosmin Petca, Department of Urology, 'Prof. Dr. Th. Burghele' Clinical Hospital, 20 Panduri Street, 050659 Bucharest, Romania

E-mail: drpetca@gmail.com

${ }^{*}$ Contributed equally

Key words: HPV vaccination, HPV transmission routes, Gardasil 9, human papillomavirus, HPV related disease initial effects: A decrease in the incidence of high-grade cervical abnormalities, no new genital warts cases in females under 21 years. Promoting greater understanding in the general public about the evident benefits of vaccination can create positive vaccine attitudes and scatter the myths of spurious side effects.

\section{Contents}

1. Introduction

2. Risk factors for HPV infection

3. Routes for HPV transmission

4. Everyday hygiene measures

5. Wart treatment

6. Prophylactic treatment of HPV-related disease

7. Campaigns for raising awareness of HPV

8. Conclusions

\section{Introduction}

Human papilloma viruses (HPVs) belong to the Papillomaviridae family and are nonenveloped double-stranded DNA viruses with over 200 types identified (1). All HPV types are epitheliotropic, infect squamous epithelia (skin and mucosae). HPV infection is widespread, in the US, the incidence is $1 \%$ in sexually active adults, and $3 \%$ in sexually active adolescents (2). Annually, 14 million persons are infected and 79 million persons have a ubiquitous infection (3).

In 2016 the estimated global prevalence of cervical HPV was $11.7 \%$ in women with non-cancerous cytology. The most frequent types worldwide are $16(3.2 \%), 18(1.4 \%), 31(0.8 \%)$ and $58(0.7 \%)$, all oncogenic papillomaviruses (4). Cervical cancer is the 4 th cause of female cancer worldwide. HPV type 16 is responsible for $50 \%$ of cervical cancer cases, and together with type 18 , account for $70 \%$ of cases (4).

HPV is estimated to be the cause of $99 \%$ of cervical cancers [there is no evidence of significant genetic predisposition for cervical cancer (5)], $90 \%$ of anal cancer, $65 \%$ vaginal cancers, $50 \%$ vulvar cancers, and 45-90\% oropharyngeal cancers (6-8). 
The microabrasions of epithelia permit the HPV particles to penetrate the basal layer of cells (the early phase probably promotes HPV-related disease) (1). The virus can remain latent or inactive for months or years (the mechanism may be immunological). Hormones are presumed to participate in the carcinogenic mechanism (5); thus, the malignancy prospect in the case of new or recurrent HPV infections in menopausal women is uncertain.

The HPV-related disease is divided into malignant and benign tumors. The virus invades its host through the anogenital and/or oropharyngeal mucosae. The effects range from warts (low-risk types) to intraepithelial dysplastic lesions, which can advance to invasive carcinoma (high-risk types). HPV spread is the source for skin, flat, or plantar warts.

The Center for Disease Control affirms that $\sim 80 \%$ of women and $90 \%$ of sexually active men, at a particular time of their life, will be infected with at least one HPV type $(4,9)$.

An anogenital verruca is the most common sexually transmitted disease in the United States: in 1\% of sexually active adults and $3 \%$ of sexually active teenagers. It affects both genders, $67 \%$ of patients being women (2). Infection with HPV types 6 and 11 predispose the appearance of anogenital warts in a person. A study states that the clinical presentation of symptoms is approximately 11-12 months among males and 5-6 months among females (4).

Most HPV infections are asymptomatic and/or they resolve spontaneously. The clearance time is $\sim 6-24$ months: Cervical HPV in 9.4 months and genital HPV in men 7.5 months (oncogenic and non-oncogenic types). Warts develop in 6-10 months after initial infection (3).

The process of cancer development from HPV infection takes $\sim 5-10$ years minimum and 20-25 years on average (10).

\section{Risk factors for HPV infection}

Multiple sexual partners; Beginning of sexual activity in the adolescence (4); Cigarette smoking: HPV infection is a decisive component for cervical malignancy, but cigarette smoking is a co-factor (local and systemic immunosuppressive effects); Oral HPV type 16 has a higher prevalence (2\%) in tobacco users (11); Sexually transmitted disease, lack or delayed treatment (12).

The International Agency for Research on Cancer (IARC) listed 7 viruses as 'carcinogenic to humans': Human T-lympho-trophic virus 1 (HTLV-1), Ebstein Barr virus (EBV), human herpes virus 8 (HHV-8), hepatitis B virus (HBV), hepatitis $\mathrm{C}$ virus (HCV), human immunodeficiency virus 1 (HIV-1) and HPV (types: 16, 18,31,33, 35,39,45,51,52,56,58 and 59) (4).It is estimated that $15-20 \%$ of cancers are attributed to viruses (9), but only a small percentage of viral infections progress to cancer with a long evolution (years or decades). Over $30 \mathrm{HPV}$ genotypes spread among the anogenital mucosa, 15 of those are liable for cervix malignancies (13).

Cervical carcinogenesis is represented by 4 causal stages (5): HPV acquisition; HPV persistence (vs. clearance); Progression to precancerous lesions; Invasive cancer.

\section{Routes for HPV transmission}

The route of HPV transmission is primarily through skin-to-skin or skin-to-mucosa contact. Sexual transmission is the most documented, but there have been studies suggesting non-sexual ways.

Horizontal transmission of $H P V$. The horizontal transmission of HPV includes fomites, fingers, and mouth, skin contact (other than sexual). These possible routes have been largely discussed in various studies. Contaminated equipment in gynecological examination rooms has been an interesting topic of evaluation in HPV infection routes.

We know about HPVs that they are very steady viruses, repellant to heat, and drying [they show 30\% infectivity after seven days of dehydration (14)]. The viruses are apt to live for days on surfaces, clothing, frequently used gynecological equipment, and fomites. Many studies have collected HPV DNA samples to assess these concerns.

A study evaluating this particular HPV transmission pattern has detected HPV DNA in 32 out of 179 (17.9\%) samples collected from gynecological equipment; $11.8 \%$ were positive in hospital compared with $27.5 \%$ in private offices, which highlights a contamination rate 2.7 times higher in private practices than a hospital. A study has also revealed that $29.4 \%$ of gloves were contaminated, and colposcopy rooms have a higher risk of being infected with HPV than outpatient offices (6).

Transvaginal ultrasound probes are protected with condoms, which act as a physical barrier to contamination (the risk of condom rupture is $\sim 0.9-5 \%$ ). Samples (21\%), after ultrasound examination (with proper disinfection), were positive for HPV DNA (15). Another study revealed, by collecting high-risk HPV DNA samples from transvaginal ultrasound, after low-level disinfection and probe cover, that a considerable number of probes were contaminated: Probes (3\%) tested positive in post-examination samples and $1.9 \%$ in pre-examination samples (14). Some studies looked even further at the samples collected from ultrasound probes and discovered not only HPV DNA but also free virions (1).

Most individuals are not aware they are infected because they are asymptomatic. Two different studies, one held in a university and one in a hospital, have revealed an abundance of HPV DNA on the fingers of infected persons, highlighting inadequate or non-existing hand hygiene (1). Studies demonstrated DNA HPV under fingernails of individuals with genital warts, as well as in their underwear (6).

Self-inoculation. Self-inoculation is described in studies as a potential HPV transmission route. It has been detailed in studies of HPV in female virgins (16), in children with genital warts (low-risk HPV) without a history of sexual abuse (17). A study on female virgins found $51.1 \%$ HPV positive, compared with $69.1 \%$ of sexually active females. Nevertheless, a survey showed that high-risk HPV is sexually transmitted, following 100 virgin females pre- and post-sexual contact $(1,4)$.

Vertical transmission. Vertical transmission from mother to newborn is another HPV transmittal route. Several studies have emphasized the possibility of infection through the amniotic fluid, or the placenta, or via contact with maternal genital mucosa during natural birth.

The possibility of vertical HPV infection was discussed as early as 1950 , in a study on infantile anogenital warts, 
Table I. Wart treatment (2).

Topical treatment

Systemic treatment

Podophyllotoxin and podophyllin (recurrence rate 38\%) - induces wart necrosis

Interferon

Imiquimod (immunomodulator)

Isotretinoin

Polyphenol E (green tea extract)

Intralesional immunotherapy (injection with candida, mumps or trichophyton skin test antigens)

Cidofovir (induces apoptosis) - not FDA approved

5-Fluorouracil - not FDA approved

Trichloroacetic acid - chemical burns, cauterizes and erodes skin and mucosa

Cryotherapy

Potassium hydroxide - dissolves keratin and penetrates the skin

Surgical excision

Photodynamic therapy

Carbon dioxide laser

Table II. HPV vaccine coverage in 2015 in Australia (24).

\begin{tabular}{llccc}
\hline Age (years) & Sex & $\begin{array}{c}\text { 1st dose } \\
(\%)\end{array}$ & $\begin{array}{c}\text { 2nd dose } \\
(\%)\end{array}$ & $\begin{array}{c}\text { 3rd dose } \\
(\%)\end{array}$ \\
\hline 14 & Girls & 87 & 85 & 79 \\
15 & Boys & 82 & 79 & 74 \\
& Girls & 86 & 83 & 78 \\
& Boys & 78 & 75 & 67 \\
\hline
\end{tabular}

Coverage for the third dose increased by 10 percentage points. $\mathrm{HPV}$, human papilloma virus.

congenital conjunctival papilloma, and juvenile laryngeal papillomatosis (18).

Another study has revealed that HPV infection prevalence among pregnant women is low (13.4\%). Among HPV types, the most frequent were type 16 (29.6\%), $18(14.7 \%)$ and $58(14.2 \%)$. Evaluated by pairs, mother-new born, the concordance in HPV DNA was $23.6 \%$. A higher prevalence of HPV DNA in female newborns $(17.7 \%)$ over males $(11.6 \%)$ was observed, without delivery-type relevance (18). This study has concluded that HPV type 16 is the most prevalent in pregnant women, and also in newborns, regardless of the nature of birth, as infection can occur during pregnancy, through the transplacental route.

Data show there is a correlation between the HPV DNA load of the mother and the ability to transfer it to newborns (1). A population-based study conducted over 20 years in Denmark found women with warts to have a 200-fold risk of their children developing juvenile laryngeal papillomatosis (1).

Waterborne transmittal. Waterbone transmittal of HPV has never been proven; still, HPV DNA has been identified in water habitats. In 2008, a study in United States concerning the variety of viruses in raw sewage uncovered for the first time 2/12 HPVs in sewage samples, but not in treated wastewater. Bibby and Peccia (19) identified in 2013 HPV in sewage sludge. They also discovered oncogenic papillomavirus: $16,18,53$,
Table III. Moderately high coverage with HPV vaccine; in Australia in school and beyond school age (26).

\begin{tabular}{lcc}
\hline Age (years) & $\begin{array}{c}\text { One-dose } \\
\text { coverage }(\%)\end{array}$ & $\begin{array}{c}\text { Three-dose } \\
\text { coverage }(\%)\end{array}$ \\
\hline $12-13$ & 83 & 73 \\
$14-15$ & 84 & 72 \\
$16-17$ & 81 & 66 \\
$18-19$ & 64 & 38 \\
$20-26$ & 52 & 32 \\
\hline
\end{tabular}

HPV, human papilloma virus.

suggesting a waterborne route of infection, as was first proposed 40 years ago (13).

Epitheliotropic viruses, as demonstrated by these studies, can be found in sewage. It might be the result of scrubbing the skin and mucous membranes, but it can also be due to urine or feces pollution $(4,13)$. Therefore, the viruses can attain water collecting sewage flow.

In Italy, Iaconelli et al, in 2015 (20) examined HPVs in two rivers and identified $56 \%$ of the samples as positive. Another study by Di Bonito et al, in 2015 (21) identified 50\% of the samples from swimming water as positive for HPV (4).

The basis for the waterborne transmission hypothesis dates from 2011 (22) when it was reported that HPV type 16 remains infectious for seven days on wet surfaces (4).

\section{Everyday hygiene measures}

Hygiene measures are proven to be inefficient in preventing HPV transmission, as the studies which have evaluated samples of HPV on contaminated medical equipment (after conventional disinfection) have found them to still be positive, especially for type 16 . Only one study has been published on HPV type 16 resistance and susceptibility to common disinfectants. HPV 16 was found resistant to alcohol-based disinfection (ethanol and isopropanol), but sensitive to hypochlorite and 
high concentrations of peracetic acid-silver-based disinfectant $(1,4)$. According to this study, we can argue for the self-inoculation and horizontal transmission of HPV. New disinfection protocols for healthcare equipment are necessary.

\section{Wart treatment}

Wart treatment involves local treatment (lesion destruction) and selective immunologic modification (Table I). No therapy has been proven to be superior in clinical clearance or recurrence.

\section{Prophylactic treatment of HPV-related disease}

In the United States, each year, 4,000 deaths occur due to cervical cancer, and more than 12,000 new cases are diagnosed. Annual costs associated with the morbidity and mortality of HPV-related diseases are estimated at $\sim \$ 4$ billion (23).

Australia initiated the first HPV vaccination program in 2007, and it lasted two years. The first prophylactic vaccine for HPV was released in 2006, covering 4 types of HPV $(16,18$ and 6,11 , which cause $90 \%$ of genital warts); in 2007, a bivalent vaccine was released, which targeted only types 16 and 18 (4). More recently, an additional vaccine has been developed, which adds 5 other types to the existing quadrivalent one: $31,33,45,52,58$; GARDASIL 9 aims to prevent $\sim 90 \%$ of HPV related disease (4).

The campaign initiated with three-dose schedule quadrivalent HPV vaccination for females aged 12-16, and lasted until the end of 2009, and routine school vaccination for 12-13-year-olds afterward. In 2013, routine vaccination began for boys up to the age of 15 (Table II) (24).

The issue of whether 2 or 3 doses are sufficient is largely studied and mostly influenced by the age of vaccine initiation (Table III).

Immunogenicity studies discovered that (5): Vaccination at young vs. old age: Higher initial, as well as subsequent antibody titers in younger people; antibody titers are higher in men than women; the difference between 2 and 3 doses is not as pronounced as the difference between 1 and 2 doses.

The World Health Organisation recommends a two-dose HPV vaccination if the first dose is initiated under 15 years of age to increase compliance and reduce the cost of vaccination [three-dose HPV vaccination is $360 \$$ at the time of data assembly (23)]. Several countries have already adopted this strategy. Coadministration of HPV vaccine with other adolescent vaccines does not decrease HPV immune responses (5). Many states cover the vaccination scheme of teenagers in one school year, as they can receive more than one vaccine a day (25).

In Australia, in 2014, there were no new genital warts cases in females under 21 years, due to the vaccine implementation $(26,27)$. There has been a decrease in genital warts in age-matched heterosexual men also, $~ 82 \%$, suggesting herd protection (5).

The early effects after the HPV vaccine program in Australia are: Decrease in the incidence of high-grade cervical abnormalities (associated with type 16 and 18), but no significant decrease in low-grade cervical abnormalities (mostly related to other types of HPV, or with inflammation) $(28,29)$. Recent data suggest a real and measurable effect of Gardasil vaccination on non-targeted HPV types.
Another benefit of vaccination could occur in people already infected with targeted types of HPV (30). There is little information on this topic, but the possibility that the antibodies could break the cycle of reinfection exists, thus reducing the residence time of infection, and also reducing the risk of transmission. Vaccinating the medical staff could have a real impact, as two laser gynecological surgeons with HPV-16 positive oropharyngeal squamous cell carcinoma were reported (31).

A study on the immunogenicity of HPV vaccines in HIV-infected females is ongoing.

The duration of protection after vaccination is not entirely known, but some trials have suggested $8-9$ years at the minimum. Seropositive persons produce higher antibodies in response to the HPV vaccine, so subsequent exposure after vaccination could serve as a booster to prolong the protection (9).

Adverse reactions of HPV vaccine, non-serious (5). Syncope, dizziness, nausea, headache, fever, hives.

A study in Melbourne on 26 out of 720 girls who, after receiving HPV vaccine in school, presented with dizziness, syncope and/or neurological symptoms, concluded these symptoms are a psychogenic response to mass vaccination in the school environment (25).

\section{Campaigns for raising awareness of HPV}

Two population-based surveys on HPV knowledge among women in Australia (before the mass media campaign on HPV vaccination) revealed modest awareness on its determinant role in cervical cancer. Only $51 \%$ of women have heard of HPV (half of whom have identified a link between HPV and cervical cancer) (25).

National surveys on HPV perception among Australians have shown that women in the middle adult years have the highest knowledge of HPV, followed by men, while the lowest awareness is among teenagers (25).

Facts revealed by the Australian studies are the 'unknown' link between HPV infection and genital warts, the fact that HPV is sexually transmitted, and that men can also be infected (25). Media campaigns presented the HPV vaccine primarily as a way to prevent cervical cancer, referring to its effects on sexually transmitted diseases (anogenital warts) as a side-note. A notable mention is the minority of parents who were reluctant to vaccinate, arguing that it can promote sexual behavior in teenagers, lacking knowledge on the subject, or being embarrassed to talk about sexually transmitted diseases with their offspring.

Promoting greater understanding in the general public about vaccination can create positive vaccine attitudes and scatter the myths of spurious side effects. By separating the health and education sectors regarding immunization, people miss the opportunity to get informed, ask questions, and understand the limitations of vaccination. Health education must be implemented in young adults, designed in such a way as to be appealing and accessible.

\section{Conclusions}

An HPV vaccination program is a long-term healthcare initiative with a high return on investment. Vaccinating all early adolescents of both genders against HPV has proven to 
be efficient and safe, and to reduce the rate of HPV related cancers and other diseases.

\section{Acknowledgements}

Not applicable.

\section{Funding}

No funding was received.

\section{Availability of data and materials}

Not applicable.

\section{Authors' contributions}

AP, RCP and MCD designed the review. AB, MEZ and FS performed the literature research and drafted the manuscript. AP, RCP and MCD substantially contributed to the conception of the study, revised and edited the final manuscript. All authors read and approved the final version of the manuscript.

\section{Ethics approval and consent to participate}

Not applicable.

\section{Patient consent for publication}

Not applicable.

\section{Competing interests}

The authors declare that they have no competing interests.

\section{References}

1. Ryndock EJ and Meyers C: A risk for non-sexual transmission of human papillomavirus? Expert Rev Anti Infect Ther 12 . 1165-1170, 2014

2. Fathi R and Tsoukas MM: Genital warts and other HPV infections: Established and novel therapies. Clin Dermatol 32: 299-306, 2014.

3. Park IU, Introcaso $\mathrm{C}$ and Dunne EF: Human papillomavirus and genital warts: A review of the evidence for the 2015 centers for disease control and prevention sexually transmitted diseases treatment guidelines. Clin Infect Dis 61 (Suppl 8): S849-S855, 2015.

4. La Rosa G: Papillomavirus. In: Global Water Pathogen Project Rose JB and Jiménez-Cisneros B (eds) (Meschke JS and Girones R (eds) Part 3 Viruses). Michigan State University, E. Lansing, MI, Unesco, 2016.

5. Castle PE and Maza M: Prophylactic HPV vaccination: Past, present, and future. Epidemiol Infect 144: 449-468, 2016.

6. Gallay C,Miranda E,Schaefer S,Catarino R, Jacot-Guillarmod M, Menoud PA, Guerry F, Achtari C, Sahli R, Vassilakos P, et al: Human papillomavirus (HPV) contamination of gynaecological equipment. Sex Transm Infect 92: 19-23, 2016.

7. Boda D, Neagu M, Constantin C, Voinescu RN, Caruntu C,Zurac S, Spandidos DA, Drakoulis N, Tsoukalas D and Tsatsakis AM: HPV strain distribution in patients with genital warts in a female population sample. Oncol Lett 12: 1779-1782, 2016.

8. You W, Li S, Du R, Zheng J and Shen A: Epidemiological study of high-risk human papillomavirus infection in subjects with abnormal cytological findings in cervical cancer screening. Exp Ther Med 15: 412-418, 2018.

9. Chesson HW, Ekwueme DU, Saraiya M, Dunne EF and Markowitz LE: The cost-effectiveness of male HPV vaccination in the United States. Vaccine 29: 8443-8450, 2011.
10. Boda D, Docea AO, Calina D, Ilie MA, Caruntu C, Zurac S, Neagu M, Constantin C, Branisteanu DE, Voiculescu V, et al: Human papilloma virus: Apprehending the link with carcinogenesis and unveiling new research avenues (Review). Int J Oncol 52: 637-655, 2018.

11. Fakhry C, Gillison ML and D'Souza G: Tobacco use and oral HPV-16 infection. JAMA 312: 1465-1467, 2014.

12. Mehedintu C, Bratila E, Cirstoiu M, Petca A, Brinduse LA, Berceanu C, Barac RI, Andreescu CV, Petca RC, Sandru F, et al: Evaluation of effectiveness and tolerability of boric acid in the treatment of vaginal infection with Candida Species. Rev Chim 70: 2375-2378, 2019.

13. Fratini M, Di Bonito P and La Rosa G: Oncogenic papillomavirus and polyomavirus in water environments: Is there a potential for waterborne transmission? Food Environ Virol 6: 1-12, 2014.

14. Casalegno JS, Le Bail Carval K, Eibach D, Valdeyron ML, Lamblin G, Jacquemoud H, Mellier G, Lina B, Gaucherand P, Mathevet $\mathrm{P}$, et al: High risk HPV contamination of endocavity vaginal ultrasound probes: An underestimated route of nosocomial infection? PLoS One 7: e48137, 2012.

15. Ma ST, Yeung AC, Chan PK and Graham CA: Transvaginal ultrasound probe contamination by the human papillomavirus in the emergency department. Emerg Med J 30: 472-475, 2013.

16. Tay SK, Ho TH and Lim-Tan SK: Is genital human papillomavirus infection always sexually transmitted? Aust NZJ Obstet Gynaecol 30: 240-242, 1990.

17. Mammas IN, Dalianis T, Doukas SG, Zaravinos A, Achtsidis V, Thiagarajan $\mathrm{P}$, Theodoridou $\mathrm{M}$ and Spandidos DA: Paediatric virology and human papillomaviruses: An update. Exp Ther Med 17: 4337-4343, 2019.

18. Hong Y, Li SQ, Hu YL and Wang ZQ: Survey of human papillomavirus types and their vertical transmission in pregnant women. BMC Infect Dis 13: 109, 2013.

19. Bibby K and Peccia J: Identification of viral pathogen diversity in sewage sludge by metagenome analysis. Environ Sci Technol 47: 1945-1951, 2013.

20. Iaconelli M, Petricca S, Libera SD, Di Bonito P and La Rosa G: First detection of human papillomaviruses and human polyomaviruses in river waters in Italy. Food Environ Virol 7: 309-315, 2015.

21. Di Bonito P, Della Libera S,Petricca S, Iaconelli M, Sanguinetti M, Graffeo R, Accardi L and La Rosa G: A large spectrum of alpha and beta papillomaviruses are detected in human stool samples. J Gen Virol 96: 607-613, 2015.

22. Ding DC, Chang YC, Liu HW and Chu TY: Long-term persistence of human papillomavirus in environments. Gynecol Oncol 121: 148-151, 2011.

23. Gostin LO: Mandatory HPV vaccination and political debate. JAMA 306: 1699-1700, 2011.

24. Brotherton JM, Winch KL, Bicknell L, Chappell G and Saville M: HPV vaccine coverage is increasing in Australia. Med J Aust 206 262, 2017.

25. Garland SM, Skinner SR and Brotherton JM: Adolescent and young adult HPV vaccination in Australia: Achievements and challenges. Prev Med 53 (Suppl 1): S29-S35, 2011.

26. Chow EP, Read TR, Wigan R, Donovan B, Chen MY, Bradshaw CS and Fairley CK: Ongoing decline in genital warts among young heterosexuals 7 years after the Australian human papillomavirus (HPV) vaccination programme. Sex Transm Infect 91: 214-219, 2015.

27. Donovan B, Franklin N, Guy R, Grulich AE, Regan DG, Ali H, Wand $\mathrm{H}$ and Fairley CK: Quadrivalent human papillomavirus vaccination and trends in genital warts in Australia: Analysis of national sentinel surveillance data. Lancet Infect Dis 11: 39-44, 2011.

28. Brotherton JM, Fridman M, May CL, Chappell G, Saville AM and Gertig DM: Early effect of the HPV vaccination programme on cervical abnormalities in Victoria, Australia: An ecological study. Lancet 377: 2085-2092, 2011.

29. Brotherton JM, Saville AM, May CL, Chappell G and Gertig DM: Human papillomavirus vaccination is changing the epidemiology of high-grade cervical lesions in Australia. Cancer Causes Control 26: 953-954, 2015.

30. Paduraru DN, Bouariu A, Ion D, Dumitrascu MC, Nitipir C, Pantea Stoian A, Hainarosie R, Bolocan A, Orlov C, Badiu DC, et al: Latest news and trends in what concerns the risk factors of endometrial cancer. Rom Biotechnol Lett 23: 14056-14066, 2018.

31. Rioux M, Garland A, Webster D and Reardon E: HPV positive tonsillar cancer in two laser surgeons: Case reports. J Otolaryngol Head Neck Surg 42: 54, 2013. 